\title{
Evaluation of an Efficient Modulus in the Biopolymer String Tension
}

\section{Vladimír Pata ${ }^{1}$, Oldřich Šuba ${ }^{1}$, Libuše Sýkorová ${ }^{1}$, Milena Kubišová1, Lenka Hýlová ${ }^{1}$, Martina Černeková ${ }^{2}$}

${ }^{1}$ Tomas Bata University in Zlín, Faculty of Technology, Department of Production Engineering, Vavrečkova 27576001 Zlín, Czech Republic

${ }^{2}$ Tomas Bata University in Zlín, Faculty of Technology, Department of Fat, Surfactant and Cosmetics Technology, Vavrečkova 27576001 Zlín, Czech Republic. Corresponding mail:*mkubisova@utb.cz

Application of biopolymer materials is currently a rising trend. However, many problems are often encountered when evaluating and describing their mechanical properties. The present article will describe a procedure for finding a so-called effective modulus of elasticity of a biopolymer material having the shape of a thin string with a diameter of about $100 \mu \mathrm{m}$.

Subsequently, there are not only problems in the actual evaluation of the tensile test (which will be demonstrated by the assembled 3D model), but also when the sample is sampled, which is decisive for finding an efficient module. The article will deal with the methodology of sample squeezing into the specially developed product, the determination of an elastic modulus from the tensile test and the behaviour of the sample when the strength limit is exceeded, which will be captured and subsequently evaluated by the high-speed camera system.

Keywords: biopolymer fibre, keratin, statistical evaluation, tensile strength

\section{Introduction}

The biopolymer fibre is made of keratin cells, with a highly organised structure. The biopolymer has the shape of an elongate cylinder whose length varies considerably. The thickness of the fibre may also vary significantly from 17 to $181 \mu \mathrm{m}$.

For testing, a biopolymer fibre was selected in the form of a hair, where it was necessary to develop a methodology for testing the properties. As the biological material is tested, the variability of the properties can be assumed, and the methodology has to be elaborated to exclude as many factors as possible to the objectivity of the test. $[1,2,12]$
In the framework of the methodology, it was necessary to define the conditions for sampling and the preservation of the specimen, the appropriate working size of the sample. The conditions for the creation of the test body, the method of measuring the thickness of the fibre and the non-corrosion control in the area of the working part, the suitable sample attachment during the tensile test. $[1,2,3,11]$

\section{Materials and Methods}

The Linear Height LH-600 B Mitutoyo was used to measure hair thickness. The hair was attached to the measuring prism of the instrument and the clamp and roller contact $\varnothing 5 \mathrm{~mm}$ was installed on the device.

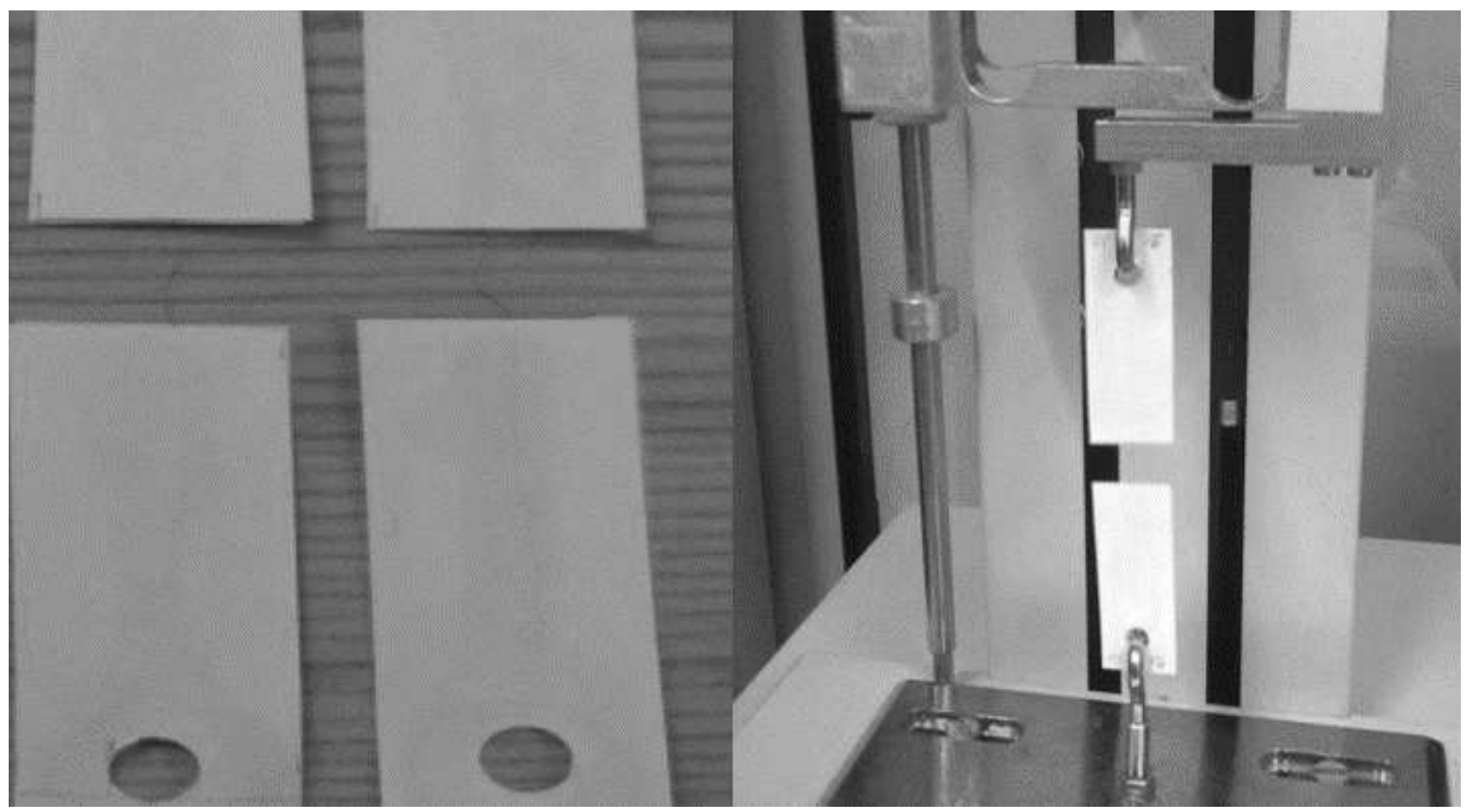

Fig. 1 Scheme of biopolymer string testing 
Also, the design of the surface treatment and the confirmation of the intact hair structure over the entire length of the work with the LEICA DMI $3000 \mathrm{M}$ microscope has been proposed for the development of the methodology. The device is primarily designed for examining the microstructure of structural materials, examining the composition of composites and evaluating the surface quality of the products. Using a microscope, the surface of all test specimens was documented before the tensile test was performed to eliminate pre-ruptured specimens. [4 - 9]

Measurements were made using a PROMI PC force measuring instrument with a capacity of $3 \mathrm{~N}$. The first experiments in the experiment were carried out with the standard clamping device supplied with the device by the manufacturer. The pulleys used to clamp the strings during testing were more appropriate than conventional jaws. The clamping of the hair without the above paper holders proved impossible, and even with the paper holders, it was challenging to attach the sample to these clamping systems in such a way that the hair was not damaged and the test was broken in the working part of the test body. The simple clamping system was finally drafted, which uses metal hooks and test body on hooks slips merely for pre-prepared holes. [5,8,10]

\subsection{Mechanical behaviour of non-homogeneous poly- mers}

Let us consider cases of uniaxial stretching of the elements that are not homogeneous, the cross-section of mechanical properties changes, whether discontinuous (due to the layered structure) or continuously. Equivalence between the axial force $\mathrm{N}$ and the internal elementary forces in the cross-section of the member is given by the equation of equilibrium.

$$
\mathrm{N}=\int_{(s)} d N=\int_{(s)} \sigma(y, z) d S,
$$

Where:

$\sigma(\mathrm{y}, \mathrm{z}) \ldots$ is the normal stress at the general point of the cross-section of the ordinates $(y, z)[\mathrm{Pa}]$

As shown in fig. 2, the beam is deformed at the axial (tensile or compressive) force load so that all of its intended longitudinal "threads" have the same proportional extension and shortening.

$$
\varepsilon(\mathrm{y}, \mathrm{z})=\varepsilon=\mathrm{constant}
$$

Where:

$\varepsilon(\mathrm{y}, \mathrm{z}) \ldots$ prolongation [unitless or $\%$ ]

Let us assume the linearly elastic behaviour of the rod material, materials of all the parts from which the member is composed. According to Hooke's law, the stress at the general point of the cross-section (assuming uniaxial strain).

$$
\sigma(\mathrm{y}, \mathrm{z})=\mathrm{E}(\mathrm{y}, \mathrm{z}) \varepsilon
$$

Where:

E... a tensile modulus $[\mathrm{Pa}]$

$$
\varepsilon=\frac{N}{\int_{(s)} E(y, z) d S}
$$

The expression in the denominator of relationship (3) is the stiffness of the tension (pressure) of the inhomogeneous, composite (composite) rod. Let us now consider a cross-section of a rod composed of several parts between which there is an unbreakable bond. Then, according to (4)

$$
\varepsilon=\frac{N}{\sum_{i=1}^{n} E i S i}=\frac{N}{E_{1}\left(s_{+}+\frac{E_{2}}{E_{+}} s_{2}+\ldots\right)}=\frac{N}{E_{1} S_{R}},
$$

The tensile stress in the elastic stress area is given for the i-th section of the cross-section

$$
\sigma_{i}=\frac{E_{i}}{E_{1}} \frac{N}{S_{R}} \text {. }
$$

Where:

$\mathrm{n}$... is the number of cross sections [-]

Ei... the elastic modulus of the i-th part [Pa]

$\mathrm{Si} . .$. area of the $\mathrm{i}$-th cross section $\left[\mathrm{mm}^{3}\right]$

SR ... reduced cross-sectional area (on the selected modulus of elasticity E1, usually E1 = $\max$ Ei) $\left[\mathrm{mm}^{3}\right]$

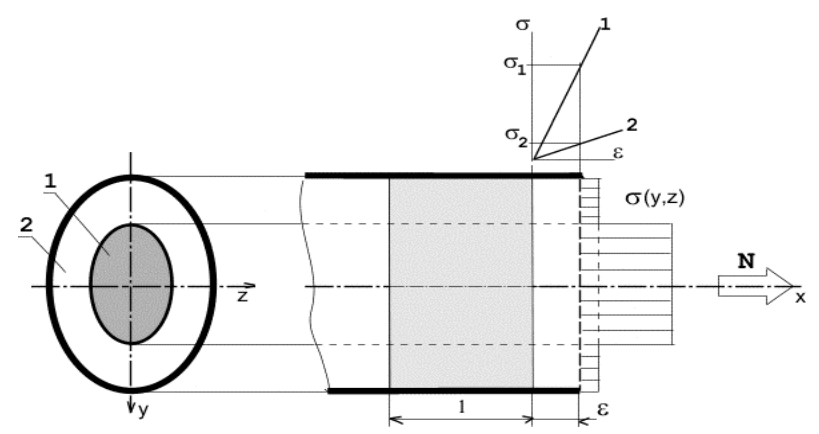

Fig. 2 Axial force load

\section{Results}

Alternative HA hypothesis: Values do not come from the basic file.

The value of the test criterion is 0.05 . If the P-value is less than this test criterion, the zero hypothesis is rejected in favour of the alternative.

From Fig. 4, it is evident that the P-value for a $10 \mathrm{~mm}$ long hair is 0.618 . We do not accept the zero hypothesis H0 with a probability of $95 \%$, the possibility of error is 5 $\%$. We can once again confirm that the data comes from a typical division.

Remote test values apply only to the normal division. As the measure of the distance of the value, its distance from the arithmetic average of the data with a normal distribution is based on the standard deviation. As shown in Fig. 5, the set of measured values of the force at the rupture has no outlying values. 


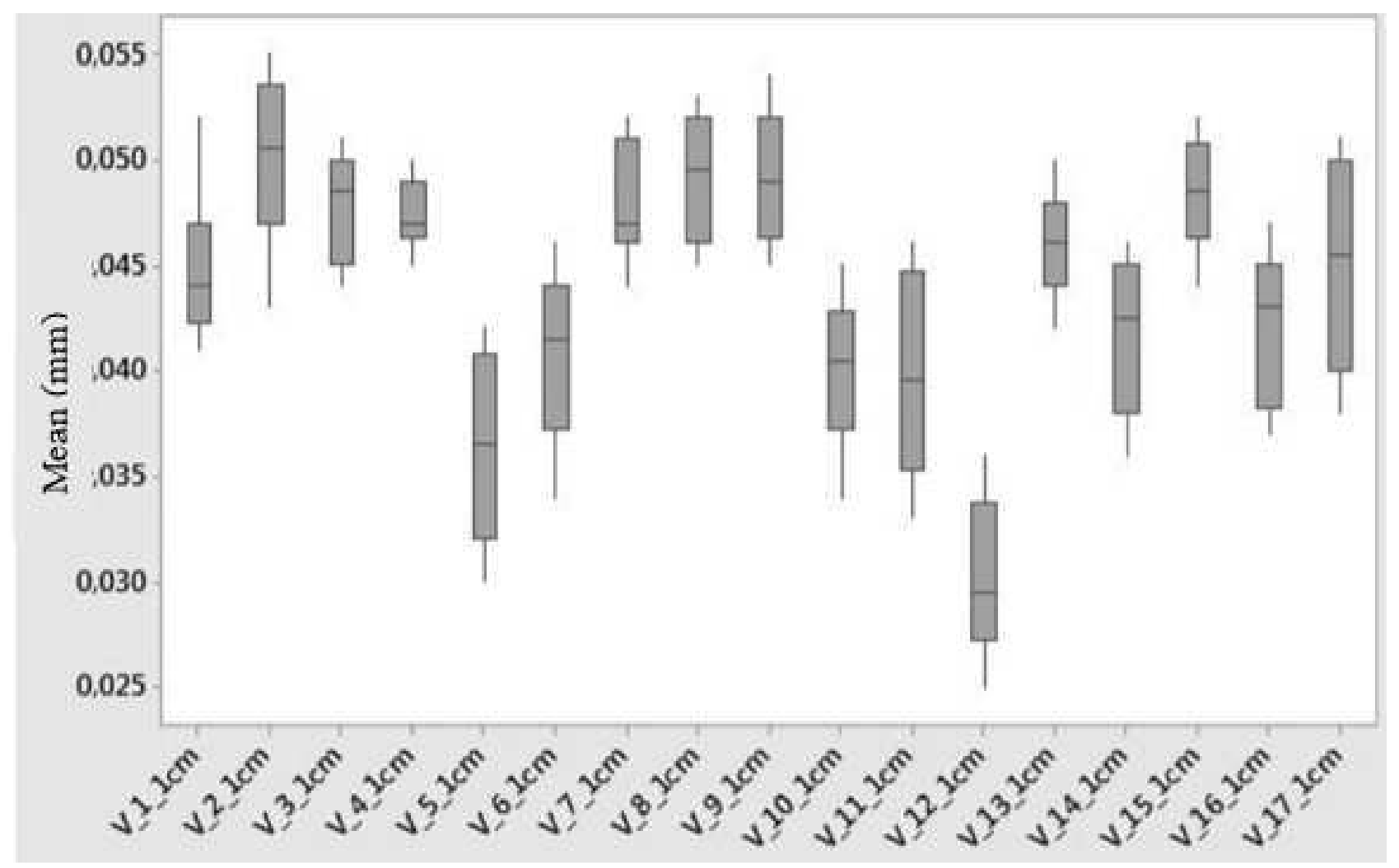

Fig. 3 Boxplot diagram

Tab. 1 Data of measurements

\begin{tabular}{|l|l|l|l|}
\hline & Units & $\begin{array}{l}\text { A sample of length 10 } \\
\text { mm } \mathbf{F}_{\max }\end{array}$ & $\begin{array}{l}\text { A sample of length 50 } \\
\text { mm } \mathbf{F}_{\max }\end{array}$ \\
\hline Arithmetic mean & $\mathrm{N}$ & 0.6512 & 0.6958 \\
\hline Standard deviation & $\mathrm{N}$ & 0.1607 & 0.1362 \\
\hline Variation coefficient & $\%$ & 24.680 & 19.570 \\
\hline Minimum & $\mathrm{N}$ & 0.3630 & 0.5700 \\
\hline First quartile $\mathbf{Q}_{\mathbf{1}}$ & $\mathrm{N}$ & 0.5350 & 0.5723 \\
\hline Median & $\mathrm{N}$ & 0.6180 & 0.6735 \\
\hline Third quartile & $\mathrm{N}$ & 0.7855 & 0.8363 \\
\hline Maximum & $\mathrm{N}$ & 0.9440 & 0.8550 \\
\hline Dispersion & $\mathrm{N}$ & 0.5810 & 0.2850 \\
\hline Quartile dispersion $\mathbf{I Q}_{\mathbf{R}}$ & $\mathrm{N}$ & 0.2505 & 0.2640 \\
\hline
\end{tabular}
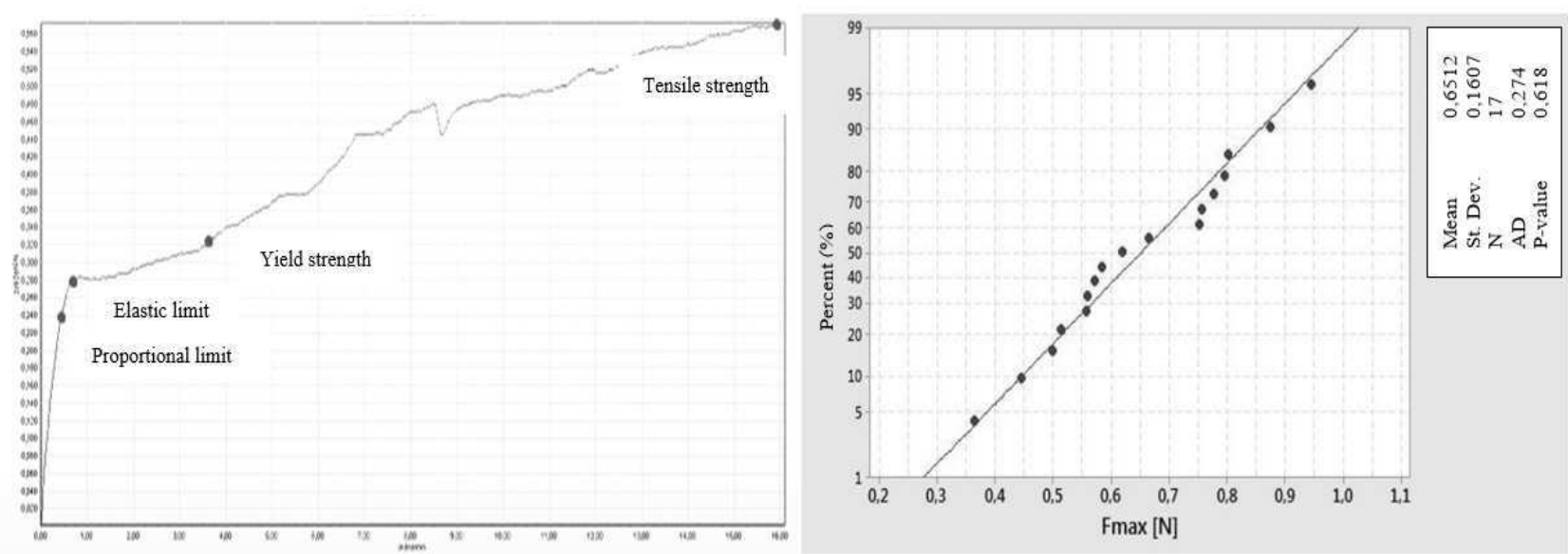

Fig. 4 Elastic modulus and Tensile strength curve 


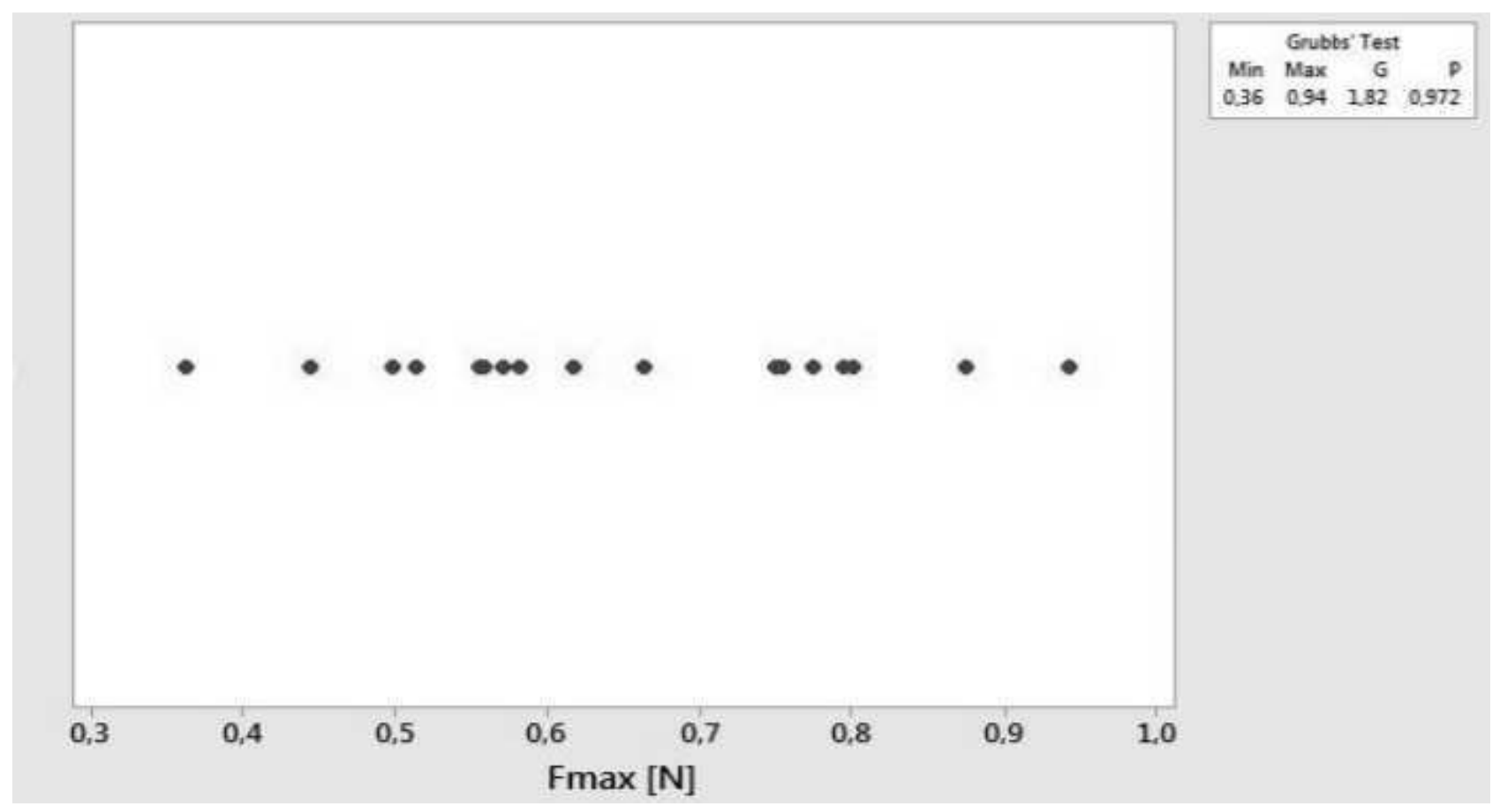

Fig. 5 Outlier test

Test of the difference between the two scattering

We test the accuracy of two measurement methods. The calculation of the F-test is based on the data of two sample sets that are being compared. Usually, this is a trial and control file.

The F-test tests a zero hypothesis that the length of the examined hair does not affect the measurement.
Where $\mathrm{F}$ is the Fischer-Snedecor division, and Fkrit is the critical value at the $5 \%$ level of significance.

Since the calculation shows that the value $\mathrm{F}<\mathrm{Fkrit}$, we can not reject the zero hypothesis. Thus, with $95 \%$ confidence, the length of the hair does not affect the test method. That is why we can work with $10 \mathrm{~mm}$ and $50 \mathrm{~mm}$ hair length.

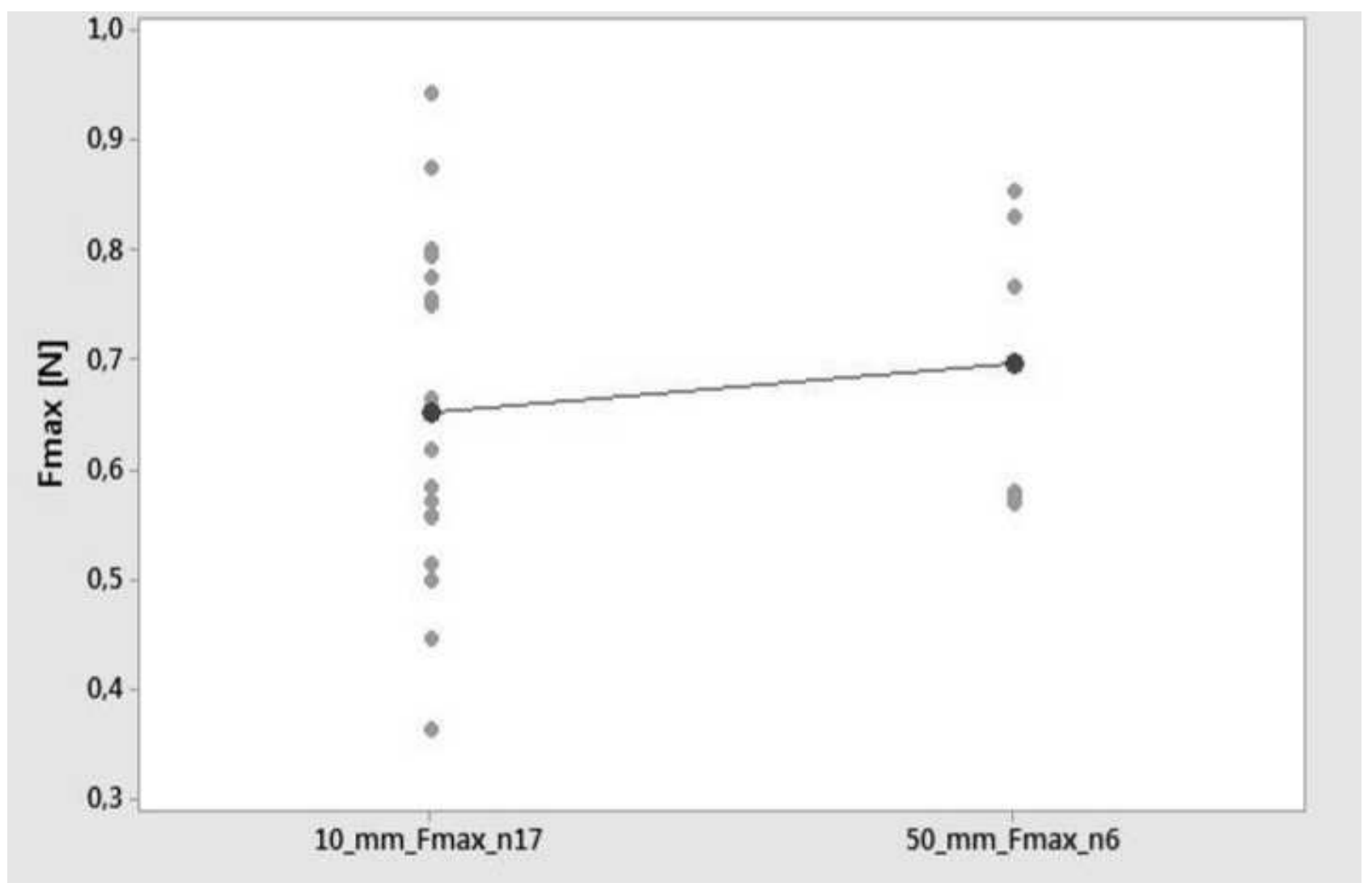

Fig. 6 Mean values of needed force to tear the biopolymer of length $10 \mathrm{~mm}$ and $50 \mathrm{~mm}$ 

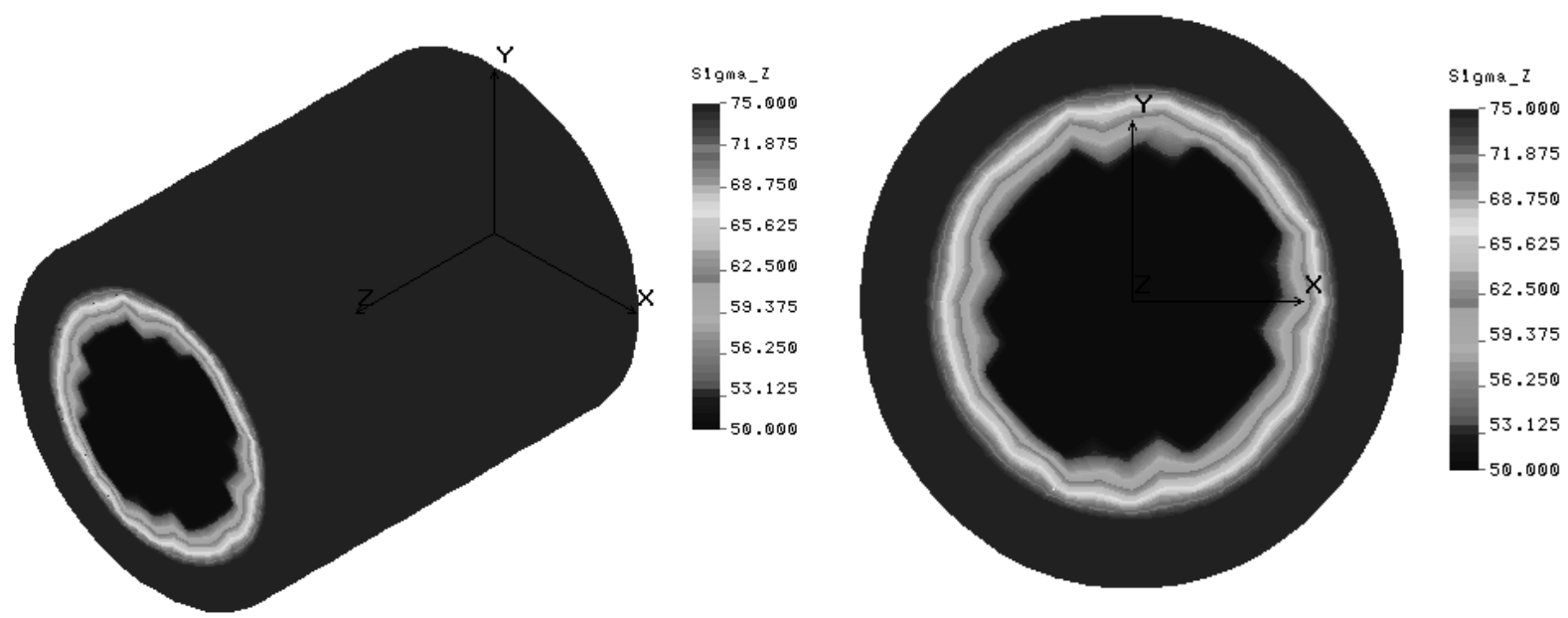

Fig. 7 The tension in the fibre corresponds to an extension of 5\%.

\section{Equal test of two mean values (t-test)}

This is one of the most used tests. The independence of selection is guaranteed by the fact that each selection contains other elements. This test is parametric and is based on the assumption that we have selections from a normal distribution.

The two-median equation test compares the data coming from subjects subjected to two measurements. Therefore, it performs two measurements per sample. Thus obtained values form pairs and represent both the control and experimental groups of the data compared to the test. The test is based on the differences of the measured pair values in the compared variation ranges. [6 - 9]

For the test of two mean values of the normal distribution, we assume that the mean values of two sets with the normal distribution from which the selection was made are equal.

Zero hypothesis H0: The mean breaking force of the $10 \mathrm{~mm}$ sample is equal to the mean breaking strength of the $50 \mathrm{~mm}$ sample.

Alternative HA hypothesis: The mean breaking strength of a $10 \mathrm{~mm}$ sample differs statistically significantly from the mean breaking strength of a $50 \mathrm{~mm}$ sample. [4 - 10]

\section{Conclusion}

The issue of biopolymer materials is now at the beginning of a separate study. The purpose of this article was to investigate the relative complexity of the problem in the case of biopolymer strings that are being studied by drawing. As a case material, a human hair with the features described in the article was selected. After choosing the appropriate mathematical apparatus, a biopolymer fibre behavioural pattern was developed during the tensile test, for which it was necessary to determine the basic and effective Young's tensile modulus.

After the model was created, it was necessary to solve the biopolymer fibre clamping process in its device having a $3 \mathrm{~N}$ force range of $0.001 \mathrm{~N}$ resolution. For this purpose, a special product has been made, and the article in- cludes a description of its handling, including the procedure itself.

The final part of the present article is the use of hypotheses theory, namely, scatter tests and mean value tests for two types of biopolymer strings having a diameter that differs statistically insignificant but having different lengths, namely $5 \mathrm{~mm}$ and $10 \mathrm{~mm}$.

In conclusion, it can be stated that for each length, the model agreed with the model, which in some cases exceeded $90 \%$. Due to the heterogeneity of the biopolymer string parameters, this result can be considered satisfactory and a promise for further research, which is developing extensively in our institute in this field.

The scientific study of biopolymer strings in recent years has gained importance. The present article is based on researches itself in this area and has taken a biopolymer string for its investigation, with its average being determined by repeated measurements at $0.02 \mathrm{~mm}$ with type A uncertainty of $0.001 \mathrm{~mm}$ - see passage 2 Materials and Methods.

To test this type of polymer string, it was suggested to use the theory of the mechanical behaviour of non-homogeneous polymers, which showed that two types of elastic modules could be expected after the tensile test. Specifically, the elastic module for the core and the elastic module for the circumferential layer which is theoretically discussed in the relations $(1-6)$.

This theoretical finding has been confirmed by our model, with the results in Fig. 7, from which it follows, its modular duality.

As the last step, measurements were made, see Fig. 1 including relevant statistical analyses. In the case of Fig. 3 and the corresponding Tab. 1 supported by the hypothesis theory, namely t-test for samples of samples having different scattering, it is clear that there is a statistically significant difference in force for samples having different lengths and also higher values of variation coefficients within sets of samples of the same length.

This statement is in full agreement with the affirmations and the results presented in [1]. Our explanation of this phenomenon of supported model and measurement is 
precisely the existence of the dual elastic modulus described above.

\section{Acknowledgement}

The authors gratefully acknowledge the financial support of this research by the internal grant of Tomas Bata University in Zlín No. IGA/FT/2018/004 and IGA/FT/2018/012 funded from the resources of specific university research.

\section{References}

[1] BOUILLON, Claude a John WILKINSON. Science of Hair Care [online]. 2nd ed. London: Taylor, ISBN 0-203-02722-1

[2] FEUGHELMAN, M. Cooperative unfolding of $\alpha$ keratin. Journal of Applied Polymer Science [online]. 1966, 10(12), 1937-1947. http://onlinelib-

rary.wiley.com/wol1/doi/10.1002/app.1966.0701

[3] MOTTRAstract. F. J. a J. R. COAD. Changes in the Cross-Linking of Keratin Fibers as Revealed by Stress-Relaxation Studies. Textile Research Journal [online]. 1974, 44(7), 557-558. http://trj.sagepub.com/content/44/7/557.full.pdf + html

[4] WHITEHOUSE, D. J., 2011. Handbook of surface and nanometrology. 2nd ed. Boca Raton: CRC Press. ISBN 978-1-4200-8201-2.

[5] BILODEAU, M. A BRENNER D. Theory of multivariate statistics. New York: Springer, c1999. Springer texts in statistics.

[6] REISS, ROLF-DIETER AND M. THOMAS. Statistical analysis of extreme values: with applications to insurance, finance, hydrology and other fields. 2nd ed. Basel: Birkhäuser Verlag, 2001. ISBN 3-7643-6487-4.

[7] BÍLEK, O. Cutting tool performance in end milling of glass fibre-reinforced polymer composites. Manufacturing Technology - Journal of Science Research and Production [online]. 2016, 16(1), 12-16 [cit. 2017-03-21]. ISSN 121-3-2489.

[8] JINFENG ZHANG, CHAO FENG, YUNHUI MA, WEI TANG, SHUAI WANG, XIN ZHONG. Non-destructive analysis of surface integrity in turning and grinding operations (2017) Manufacturing Technology, 17/3, pp. 412-418.

[9] HANZL P., ZETKOVÁ I., MACH J. Optimization of the Pressure Porous Sample and Its Manufacturability by Selective Laser Melting (2017) Manufacturing Technology, 17/1, pp. 3438.

[10] JAN PODANÝ, ALEXEY MOLOTOVNIK. 3D Measurement of Surface Texture Parameters (2014) Manufacturing Technology, 14/4, pp. 596600.

[11] MANAS, D., OVSIK, M., MIZERA, A., MANAS, M., HYLOVA, L., BEDNARIK, M., STANEK, M. The effect of irradiation on mechanical and thermal properties of selected types of polymers (2018) Polymers, 10 (2), art. no. 158.

[12] OVSIK, M., SENKERIK, V., MANAS, D., STANEK, M. The behaviour of cross-linking filled PBT measured by nano-hardness (2016) MM Science Journal, 2016 (October), pp. 11101113. 